\title{
VITAL CAPACITY OF THE LUNGS OF CHILDREN *
}

\author{
PAUL W. EMERSON
}

Junior Assistant Visiting Physician, Children's Hospital, Visiting Physician to the Boston Floating Hospital, Assistant in Pediatrics at Harvard Medical School

and

HYMAN GREEN

Junior Assistant Visiting Physician to Children's Hospital BOSTON

The vital capacity of the lungs is the volume of air which can be expelled after the deepest possible inhalation. We have been unable to find any recorded measurements of vital capacity in normal children, and this was one reason for making these determinations. West ${ }^{2}$ has shown that vital capacity in adults varies with the surface area more closely than with any other standard tried. It was not our object to determine the truth of this relation in normal children, but it came out in our work. Peabody ${ }^{1}$ has demonstrated the importance of the reduction in vital capacity as an index of the tendency to dyspnea in cardiac disease and has emphasized the importance of vital capacity determinations in diagnosis, prognosis and treatment of vital capacity. It is our primary object to confirm, with regard to children, Peabody's observations in adults, but naturally the vital capacity of normal children had first to be determined. We have made a number of measurements in children in the Heart Clinic of the Children's Hospital, and these we shall publish in a later communication.

METHOD USED

We used for all our determinations the Standard spirometer, made by the Narragansett Machine Co., Providence, R. I. With individual sterilized mouthpieces, each child was coached to take the fullest possible inspiration and then to put forth his best effort in making the maximum expiration. No determination was considered satisfactory unless two determinations came within a margin of 20 c.c. Vital capacity is, of course, the maximum expiration, but we felt that each determination should be checked; two measurements differing more than 20 c.c. from one another would be open to the criticism that the higher figure was

* Received for publication, Feb. 22, 1921.

1. Peabody, F. W., and Wentworth, J. A.: Clinical Studies of the Respiration. IV, Arch. Int. Medicine 20:443 (Oct.) 1917.

2. West, H. F.: Clinical Studies of the Respiration. VI, Arch. Int. Med. $25: 231$ (March) 1920 . 
due to two expirations instead of one expiration, or that the lower figure was due to a poor try. In a number of cases we have determinations in the same children at intervals of a week, repeated at seven or eight successive periods, and these results were practically constant. Proper coaching of the children and experience are of the greatest importance in securing reliable results. Children below seven years do not cooperate well.

\section{SOURCES OF OUR MATERIAL}

Boys between 8 and 15 years of age and girls between 7 and 13 years, inclusive, of the class of people who would attend an outpatient clinic, of various nationalities-Syrian, Irish, American, Italian and Jewish-were measured. These measurements were made in the outpatient departments of the Children's Hospital and of the Boston - Floating Hospital, at a boys' club, at an orphan asylum, and at two settlement houses. Each child was examined physically to rule out any children with cardiac or pulmonary disease. Each child was weighed stripped, and measured as to height without shoes. The number of children accepted was 413 boys and girls. Of these, sixty-three were excluded on account of inability to cooperate on account of age. There remained 350 cases, 266 boys and eighty-four girls.

We used the revised tables of Wood ${ }^{3}$ for our standard for weights and for heights. The weights as given by Wood include indoor clothes, and since our children were all weighed stripped, it was necessary in determining those cases below normal weight for height, to deduct a certain percentage of the total weight to allow for this factor. In the tables published by Dr. J. L. Morse, ${ }^{4} 7$ per cent. of the total weight for boys is allowed for indoor clothes, and 8 per cent. for girls. For convenience, we allowed 7.5 per cent. for both boys and girls. It is held by Emerson and Manny " that the zone of health, as allowed for by Wood, that is, the difference between the obese and the malnourished, is too narrow. A more reasonable zone would allow the deduction of 7 per cent. from the average weight, to include children to this extent under weight for height. These could still be considered normal. For convenience we used the factor 7.5 per cent., making a total deduction of 15 per cent. to be made from the figures of Wood. It may be pointed out that 7.5 per cent. is too much to allow as a reduction from the figures of Wood because this would put the lower boundary of the zone of health below that of Emerson and Manny, even though Wood's curve follows very closely that of Boas and Burk, set forward six months by Emerson and Manny and used by them as

3. Wood, T. D.: Right Height and Weight for Boys and for Girls. Child Health Organization.

4. Morse, J. L.: Case Histories in Pediatrics.

5. Emerson, W. R. P., and Manny, F. A.: Weight and Height in Relation to Malnutrition. Arch. Pediat. 37:468 (Aug.) 1920. 
TABlE 1.-Vital Capacity, Weight and Surface Areas of Boys

\begin{tabular}{|c|c|c|c|c|}
\hline $\begin{array}{l}\text { Height, } \\
\text { Inches }\end{array}$ & $\begin{array}{l}\text { Age, } \\
\text { Years }\end{array}$ & $\begin{array}{l}\text { Weight, } \\
\text { Pounds }\end{array}$ & $\begin{array}{c}\text { Vital Capacity, } \\
\text { C.c. }\end{array}$ & $\begin{array}{l}\text { Surface Area, } \\
\text { Sq. M. }\end{array}$ \\
\hline $\begin{array}{l}69.8 \\
67 \\
66.9 \\
66.6 \\
65.1 \\
65 \\
64.8 \\
64.4 \\
63.4 \\
63 \\
63 \\
63 \\
62.9 \\
62.7 \\
62.6 \\
62.6 \\
62.2 \\
62 \\
61 \\
61 \\
61 \\
61 \\
60.5 \\
60.5 \\
60.5 \\
60.5 \\
60.4 \\
60.2 \\
60 \\
60 \\
59.7 \\
59.7 \\
59.6 \\
59.4 \\
59.4 \\
59.4 \\
59.4 \\
59.3 \\
59.2 \\
59.2 \\
59 \\
59 \\
59 \\
59 \\
58.9 \\
58.8 \\
58.6 \\
58.5 \\
58.5 \\
58.5 \\
58.5 \\
58.4 \\
58.3 \\
58.1 \\
58.1 \\
58.1 \\
58 \\
58 \\
58 \\
58 \\
58 \\
57.9 \\
57.8 \\
57.7 \\
57.6 \\
57.6 \\
57.5 \\
57.5 \\
57.5 \\
57.3 \\
57.3 \\
57.2 \\
57.1 \\
57.1 \\
57 \\
57 \\
57 \\
57 \\
56.9 \\
56.8 \\
56.7 \\
56.5 \\
56.5 \\
56.5 \\
56.5 \\
56.5 \\
56.5 \\
56.4 \\
56.1\end{array}$ & $\begin{array}{l}15 \\
13 \\
13 \\
14 \\
15 \\
15 \\
14 \\
14 \\
14 \\
15 \\
13 \\
13 \\
15 \\
15 \\
14 \\
12 \\
15 \\
15 \\
15 \\
14 \\
14 \\
13 \\
13 \\
13 \\
13 \\
11 \\
14 \\
13 \\
13 \\
15 \\
14 \\
13 \\
14 \\
13 \\
13 \\
14 \\
15 \\
15 \\
13 \\
15 \\
14 \\
13 \\
12 \\
14 \\
15 \\
13 \\
14 \\
13 \\
13 \\
12 \\
14 \\
11 \\
13 \\
13 \\
12 \\
13 \\
13 \\
13 \\
13 \\
15 \\
13 \\
13 \\
13 \\
12 \\
14 \\
13 \\
12 \\
14 \\
10 \\
13 \\
14 \\
14 \\
13 \\
13 \\
14 \\
13 \\
15 \\
14 \\
13 \\
13 \\
12 \\
12 \\
12\end{array}$ & $\begin{array}{c}132 \\
76^{*} \\
79.6^{*} \\
138.8 \\
115.2^{*} \\
129 \\
119.5 \\
110.2 \\
125.7 \\
93 \\
121 \\
88^{*} \\
98.6 \\
101 \\
102.7 \\
97 \\
90 \\
91 \\
92 \\
102.8 \\
96 \\
108 \\
94 \\
100 \\
94 \\
95 \\
101.4 \\
100 \\
97 \\
89 \\
85.6^{*} \\
87.5 \\
108 \\
85 \\
90 \\
86.1 \\
88.7 \\
93.6^{*} \\
87.5 \\
108.5^{*} \\
75 \\
93 \\
73^{*} \\
89 \\
88.1 \\
85.3 \\
87.3 \\
88 \\
84 \\
79.7^{*} \\
771^{*} \\
90.1 \\
82.7 \\
86.5 \\
80.7 \\
77.9^{*} \\
79 * \\
96.5 \\
82.9 \\
88 \\
71^{*} \\
95.8^{*} \\
82.5 \\
85.5 \\
84.9 \\
83 \\
73.5^{*} \\
78^{*} \\
85.7 \\
76.7^{*} \\
70.8^{*} \\
73.4^{*} \\
67.2^{*} \\
81.1 \\
90 \\
95 \\
92 \\
83 \\
79.1 \\
74.5^{*} \\
77.1^{*} \\
79.5^{*} \\
83.5^{*} \\
81.2 \\
73.8^{*} \\
75 * \\
69 * \\
80.4 \\
86.5 \\
\end{array}$ & $\begin{array}{l}2,220 \\
1,300 \\
1,260 \\
2,220 \\
1,920 \\
2,200 \\
2,260 \\
2,100 \\
2,180 \\
1,600 \\
1,900 \\
1,960 \\
1,600 \\
1,800 \\
1,340 \\
1,680 \\
1,300 \\
1,400 \\
1,300 \\
1,800 \\
1,400 \\
1,680 \\
1,160 \\
1,120 \\
1,420 \\
720 \\
1,380 \\
1,600 \\
1,380 \\
1,400 \\
1,400 \\
1,160 \\
1,340 \\
1,240 \\
1,480 \\
1,560 \\
1,600 \\
2,240 \\
1,500 \\
1,500 \\
1,100 \\
1,320 \\
1,520 \\
1,440 \\
1,660 \\
900 \\
1,500 \\
1,560 \\
1,600 \\
1,400 \\
1,220 \\
1,360 \\
1,080 \\
1,340 \\
1,220 \\
1,280 \\
1,540 \\
1,360 \\
1,560 \\
1,580 \\
1,120 \\
1,260 \\
1,400 \\
1,500 \\
1,420 \\
1,300 \\
1,020 \\
1,200 \\
1,400 \\
1,360 \\
1,080 \\
1,220 \\
1,140 \\
1,260 \\
1,220 \\
1,440 \\
1,340 \\
1,320 \\
1,100 \\
1,020 \\
1,100 \\
1,420 \\
800 \\
1,100 \\
1,320 \\
1,100 \\
840 \\
1,440 \\
1,120 \\
\end{array}$ & 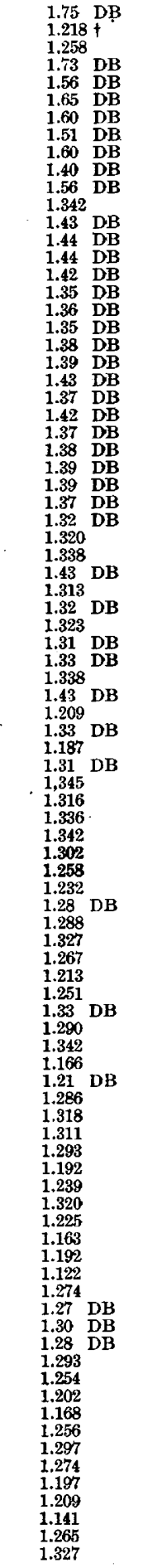 \\
\hline
\end{tabular}

* Denotes those cases falling below $\mathbf{7 . 5}$ per cent. normal weight for height.

$\dagger$ All surface areas, except those marked $D B$, are from Benedict and Talbot. DB denotes Dubois and Dubois. 
Table 1.-Vital Capacity, Weight and Surface

AREAS OF BoYs-(Continued)

\begin{tabular}{|c|c|c|c|c|}
\hline $\begin{array}{l}\text { Height, } \\
\text { Inches }\end{array}$ & $\begin{array}{l}\text { Age, } \\
\text { Years }\end{array}$ & $\begin{array}{l}\text { Weight, } \\
\text { Pounds }\end{array}$ & $\begin{array}{l}\text { Vital Capacity, } \\
\text { C.c. }\end{array}$ & $\begin{array}{l}\text { Surface Area, } \\
\text { Sq. M. }\end{array}$ \\
\hline $\begin{array}{l}56.1 \\
56 \\
56 \\
56 \\
56 \\
56 . \\
56 \\
56 \\
56 \\
56 \\
55.9 \\
55.8 \\
55.8 \\
55.8 \\
55.7 \\
55.6 \\
55.5 \\
55.5 \\
55.5 \\
55.4 \\
55.4 \\
55.4 \\
55.4 \\
55.3 \\
55.3 \\
55.1 \\
55.1 \\
55.1 \\
55.1 \\
55 \\
55 \\
55 \\
55 . \\
55 \\
55 \\
55 \\
55 \\
55 \\
55 \\
55 \\
55 \\
54.9 \\
54.8 \\
54.8 \\
54.7 \\
54.7 \\
54.7 \\
54.7 \\
54.7 \\
54.7 \\
54.6 \\
54.5 \\
54.5 \\
54.4 \\
54.4 \\
54.2 \\
54 \\
54 \\
54 \\
54 \\
54 \\
54 \\
54 \\
54 \\
54 \\
54 \\
54 \\
53.8 \\
53.7 \\
53.7 \\
53.7 \\
55.6 \\
53.5 \\
53.5 \\
53.5 \\
53.4 \\
53.2 \\
53 \\
53 \\
53 \\
53 \\
53 \\
53 \\
53 \\
53 \\
53 \\
53 \\
\end{array}$ & $\begin{array}{l}11 \\
15 \\
13 \\
13 \\
13 \\
12 \\
12 \\
12 \\
12 \\
11 \\
11 \\
12 \\
12 \\
12 \\
13 \\
12 \\
13 \\
11 \\
10 \\
14 \\
12 \\
11 \\
11 \\
11 \\
11 \\
12 \\
11 \\
11 \\
10 \\
12 \\
12 \\
12 \\
12 \\
12 \\
12 \\
11 \\
11 \\
11 \\
11 \\
11 \\
10 \\
13 \\
11 \\
11 \\
12 \\
12 \\
10 \\
10 \\
9 \\
11 \\
13 \\
13 \\
12 \\
12 \\
10 \\
11 \\
12 \\
12 \\
12 \\
12 \\
12 \\
12 \\
11 \\
11 \\
10 \\
13 \\
9 \\
13 \\
12 \\
11 \\
12 \\
13 \\
14 \\
12 \\
12 \\
11 \\
10 \\
12 \\
12 \\
12 \\
12 \\
11 \\
11 \\
11 \\
11 \\
11\end{array}$ & $\begin{array}{l}80.3 \\
82.3 \\
76 \\
75.6 \\
73^{*} \\
75 \\
73^{*} \\
76 \\
69^{*} \\
71^{*} \\
90 \\
80.3 \\
66.9^{*} \\
62.7^{*} \\
73.7 \\
77.3 \\
75.5 \\
85.7 \\
67.2^{*} \\
76.1 \\
65.1^{*} \\
71.5 \\
70.2 \\
67.5^{*} \\
72 \\
73.1 \\
77.6 \\
70 \\
65.6^{*} \\
69^{*} \\
65^{*} \\
66^{*} \\
67.7^{*} \\
74 \\
72.5 \\
74 \\
633^{*} \\
67.5^{*} \\
73 \\
70 \\
70 \\
76.5 \\
68.6^{*} \\
70.8 \\
72.3 \\
71.5 \\
64.6^{*} \\
67.5^{*} \\
69.1 \\
63.3^{*} \\
68.2^{*} \\
73.5 \\
59.1^{*} \\
66.5^{*} \\
67.4 \\
66.6 \\
64^{*} \\
68.3 \\
72 \\
69 \\
64^{*} \\
69 \\
75 \\
80 \\
64^{*} \\
69 \\
68.7 \\
66.7^{*} \\
666^{*} \\
64.4^{*} \\
67^{*} \\
60^{*} \\
66^{*} \\
70.3 \\
66^{*} \\
67.5 \\
66.9 \\
69.2 \\
66 \\
68 \\
77 \\
68.6 \\
60^{*} \\
63^{*} \\
80 \\
78 \\
65 \\
63.3^{*} \\
6\end{array}$ & 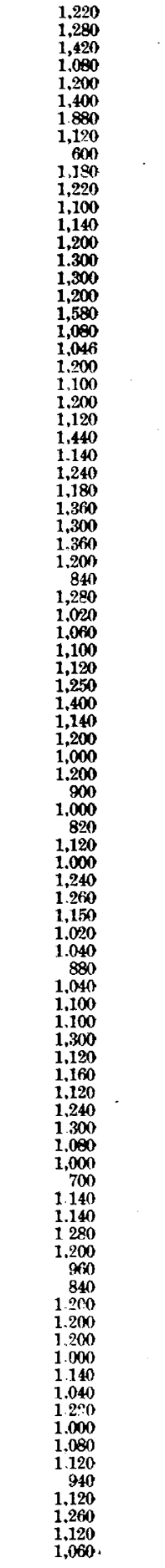 & $\begin{array}{l}1.263 \\
1.283 \\
1.218 \\
1.216 \\
1.187 \\
1.209 \\
1.187 \\
1.218 \\
1.141 \\
1.166 \\
1.26 \\
1.263 \\
1.117 \\
1.070 \\
1.195 \\
1.232 \\
1.213 \\
1.320 \\
1.122 \\
1.220 \\
1.100 \\
1.171 \\
1.156 \\
1.124 \\
1.175 \\
1.190 \\
1.237 \\
1.154 \\
1.105 \\
1.141 \\
1.098 \\
1.107 \\
1.126 \\
1.197 \\
1.180 \\
1.197 \\
1.075 \\
1.124 \\
1.187 \\
1.154 \\
1.154 \\
1.223 \\
1.136 \\
1.163 \\
1.179 \\
1.171 \\
1.093 \\
1.124 \\
1.144 \\
1.078 \\
1.131 \\
1.192 \\
1.032 \\
1.112 \\
1.124 \\
1.114 \\
1.086 \\
1.131 \\
1.175 \\
1.141 \\
1.086 \\
1.141 \\
1.209 \\
1.260 \\
1.086 \\
1.141 \\
1.136 \\
1.114 \\
1.107 \\
1.090 \\
1.119 \\
1.040 \\
1.107 \\
1.156 \\
1.107 \\
1.124 \\
1.117 \\
1.144 \\
1.107 \\
1.199 \\
1.230 \\
1.136 \\
1.040 \\
1.075 \\
1.260 \\
1.239 \\
1.098 \\
1.078 \\
\\
\\
\\
\\
\\
\end{array}$ \\
\hline
\end{tabular}

* Denotes those cases falling below 7.5 per cent. normal weight for height.

All surface areas, except those marked DB, are from Benedict and Talbot. DB denotes Dubois and Dubois. 
TABle 1.-Vital Capacity, Weight and Surface

AREAS OF Boys-(Continued)

\begin{tabular}{|c|c|c|c|c|}
\hline $\begin{array}{l}\text { Height, } \\
\text { Inches }\end{array}$ & $\begin{array}{l}\text { Age, } \\
\text { Years }\end{array}$ & $\begin{array}{l}\text { Weight, } \\
\text { Pounds }\end{array}$ & $\begin{array}{l}\text { Vital Capacity, } \\
\text { C.c. }\end{array}$ & $\begin{array}{l}\text { Surface Area, } \\
\text { Sq. M. }\end{array}$ \\
\hline $\begin{array}{ll} & 58 \\
53 \\
53.8 \\
\mathbf{5 2 . 7} \\
\mathbf{5 2 . 7} \\
\mathbf{5 2 . 7} \\
\mathbf{5 2 . 6} \\
\mathbf{5 2 . 6} \\
\mathbf{5 2 . 5} \\
\mathbf{5 2 . 4} \\
\mathbf{5 2 . 4} \\
\mathbf{5 2 . 2} \\
\mathbf{5 2 . 2} \\
\mathbf{5 2 . 1} \\
\mathbf{5 2 . 1} \\
\mathbf{5 2} \\
\mathbf{5 2} \\
\mathbf{5 2} \\
\mathbf{5 2} \\
\mathbf{5 2} \\
\mathbf{5 2} \\
\mathbf{5 2} \\
\mathbf{5 2} \\
\mathbf{5 2} \\
\mathbf{5 2} \\
\mathbf{5 2} \\
\mathbf{5 2} \\
\mathbf{5 1 . 9} \\
\mathbf{5 1 . 8} \\
\mathbf{5 1 . 8} \\
\mathbf{5 1 . 7} \\
\mathbf{5 1 . 6} \\
\mathbf{5 1 . 6} \\
\mathbf{5 1 . 5} \\
\mathbf{5 1 . 4} \\
\mathbf{5 1 . 3} \\
\mathbf{5 1 . 1} \\
\mathbf{5 1} \\
\mathbf{5 1} \\
\mathbf{5 1} \\
\mathbf{5 1} \\
\mathbf{5 1} \\
\mathbf{5 1} \\
\mathbf{5 1} \\
\mathbf{5 1} \\
\mathbf{5 1} \\
\mathbf{5 0 . 7} \\
\mathbf{5 0 . 6} \\
\mathbf{5 0 . 5} \\
\mathbf{5 0 . 5} \\
\mathbf{5 0 . 5} \\
\mathbf{5 0 . 4} \\
\mathbf{5 0 . 3} \\
\mathbf{5 0} \\
\mathbf{5 0} \\
\mathbf{5 0} \\
\mathbf{5 0} \\
\mathbf{5 0} \\
\mathbf{5 0} \\
\mathbf{5 0} \\
\mathbf{4 9 . 9} \\
\mathbf{4 9 . 8} \\
\mathbf{4 9 . 7} \\
\mathbf{4 9 . 4} \\
\mathbf{4 9 . 3} \\
\mathbf{4 9} \\
49 \\
\mathbf{4 9} \\
\mathbf{4 9} \\
\mathbf{4 9} \\
\mathbf{4 9} \\
\mathbf{4 8 . 7} \\
\mathbf{4 8 . 6} \\
\mathbf{4 8 . 5} \\
\mathbf{4 8 . 5} \\
\mathbf{4 8 . 5} \\
\mathbf{4 8 . 4} \\
\mathbf{4 8 . 3} \\
\mathbf{4 8 . 2} \\
\mathbf{4 8 . 1} \\
\mathbf{4 8} \\
\mathbf{4 7 . 6} \\
\mathbf{4 7 . 5} \\
\mathbf{4 7 . 2} \\
\mathbf{4 7} \\
\mathbf{4 6 . 7} \\
\mathbf{4 6 . 7} \\
\mathbf{4 6 . 7} \\
\mathbf{4 6 . 2} \\
\mathbf{4 6 . 1}\end{array}$ & 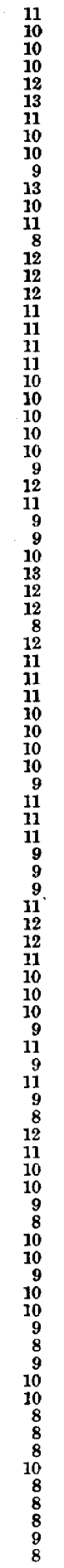 & $\begin{array}{l}68 \\
63 \\
70.5 \\
68.3 \\
58.1 \\
68 \\
67.9 \\
60.5^{*} \\
66 \\
56.5^{*} \\
55.2^{*} \\
66.8 \\
66.5 \\
53 \\
63 \\
61 . \\
58^{*} \\
58^{*} \\
59^{*} \\
68 \\
70.5 \\
63 \\
63.6 \\
65 \\
62 \\
77 \\
61 \\
68.1 \\
63.6 \\
50.4^{*} \\
54.3^{*} \\
61 \\
61.5^{*} \\
55.6^{*} \\
86 \\
59.8 \\
66 \\
58^{*} \\
511^{*} \\
61 \\
57.6^{*} \\
56 \\
60.5 \\
61 \\
65 \\
56.8^{*} \\
59.5 \\
558^{*} \\
52.2 \\
60 \\
58.2 \\
57.4 \\
58.1 \\
65 \\
72 \\
62 \\
57 \\
59 \\
55^{*} \\
53.5^{*} \\
65.8 \\
56.4^{*} \\
55.2 \\
52.3 \\
58^{*} \\
48^{*} \\
50.5^{*} \\
58 \\
54.6 \\
51.9 \\
49^{*} \\
55.3 \\
49^{*} \\
61 \\
60.8 \\
57 \\
48.3 \\
52.2^{*} \\
51.1 \\
47^{*} \\
45.3^{*} \\
53^{*} \\
46.6^{*} \\
48.5^{*} \\
48 \\
46.1^{*} \\
52.1 \\
50.7 \\
49 \\
49\end{array}$ & $\begin{array}{r}1,140 \\
1,000 \\
1,260 \\
1,100 \\
1,160 \\
1,200 \\
1,200 \\
800 \\
1,060 \\
1,200 \\
1,140 \\
920 \\
1,000 \\
900 \\
620 \\
840 \\
980 \\
1,020 \\
920 \\
1,000 \\
1,100 \\
1,000 \\
1,000 \\
1,240 \\
940 \\
860 \\
940 \\
1,060 \\
1,020 \\
1,100 \\
880 \\
820 \\
1,100 \\
1,040 \\
840 \\
900 \\
800 \\
1,020 \\
1,020 \\
780 \\
960 \\
980 \\
1,100 \\
660 \\
1,100 \\
900 \\
920 \\
1,000 \\
980 \\
1,000 \\
940 \\
1,000 \\
750 \\
1,000 \\
920 \\
720 \\
800 \\
900 \\
1,100 \\
860 \\
900 \\
820 \\
980 \\
800 \\
820 \\
840 \\
860 \\
800 \\
820 \\
820 \\
800 \\
900 \\
620 \\
1,100 \\
720 \\
800 \\
600 \\
820 \\
680 \\
720 \\
800 \\
760 \\
540 \\
800 \\
640 \\
820 \\
620 \\
820 \\
720\end{array}$ & $\begin{array}{l}1.129 \\
1.075 \\
1.159 \\
1.131 \\
1.019 \\
1.129 \\
1.129 \\
1.045 \\
1.107 \\
0.988 \\
1.082 \\
1.117 \\
1.112 \\
0.934 \\
1.075 \\
1.052 \\
1.016 \\
1.016 \\
1.029 \\
1.129 \\
1.159 \\
1.075 \\
1.083 \\
1.098 \\
1.062 \\
1.230 \\
1.052 \\
1.131 \\
1.083 \\
0.903 \\
0.947 \\
1.052 \\
1.057 \\
0.973 \\
1.323 \\
1.040 \\
1.107 \\
1.016 \\
0.911 \\
1.052 \\
1.014 \\
0.978 \\
1.045 \\
1.052 \\
1.098 \\
0.998 \\
1.035 \\
1.016 \\
0.924 \\
1.040 \\
1.019 \\
1.011 \\
1.019 \\
1.098 \\
1.175 \\
1.062 \\
1.003 \\
1.029 \\
0.958 \\
0.939 \\
1.062 \\
0.988 \\
0.963 \\
0.924 \\
1.016 \\
0.871 \\
0.903 \\
1.016 \\
0.952 \\
0.921 \\
0.884 \\
0.963 \\
0.884 \\
1.052 \\
1.050 \\
1.003 \\
0.873 \\
0.924 \\
0.913 \\
0.860 \\
0.839 \\
0.934 \\
0.855 \\
0.876 \\
0.871 \\
0.850 \\
0.924 \\
0.906 \\
0.884 \\
\end{array}$ \\
\hline
\end{tabular}

* Denotes those cases falling below 7.5 per cent. normal weight for height.

$t$ All surface areas, except those marked DB, are from Benedict and Talbot. DB denotes Dubois and Dubois. 
Table 2.-Weight, Vital Capacity and Surface Area of Girls

\begin{tabular}{|c|c|c|c|c|}
\hline $\begin{array}{c}\text { Height, } \\
\text { Inches }\end{array}$ & $\begin{array}{l}\text { Age, } \\
\text { Years }\end{array}$ & $\begin{array}{l}\text { Weight, } \\
\text { Pounds }\end{array}$ & $\begin{array}{l}\text { Vital Capacity, } \\
\text { C.e. }\end{array}$ & $\begin{array}{l}\text { Surface Area, } \\
\text { Sq. M. }\end{array}$ \\
\hline $\begin{array}{l}59.5 \\
59.3 \\
59 \\
59 \\
58.8 \\
58.8 \\
58.3 \\
58 \\
58 \\
57.8 \\
57.8 \\
57.5 \\
57 \\
57 \\
57 \\
57 \\
57 \\
56.5 \\
56.5 \\
56.3 \\
56 \\
56 \\
55 \\
55 \\
55 \\
55 \\
54.8 \\
54 \\
53.5 \\
53.5 \\
53.5 \\
53.3 \\
53.3 \\
53 \\
52.5 \\
52.5 \\
52 \\
52 \\
52 \\
51.8 \\
51 \\
51 \\
50.5 \\
50 \\
50 \\
50 \\
50 \\
50 \\
50 \\
50 \\
49.5 \\
49.1 \\
49 \\
49 \\
49 \\
49 \\
49 \\
48.5 \\
48.3 \\
48.3 \\
48 \\
48 \\
48 \\
48 \\
48 \\
47.8 \\
47.5 \\
47.5 \\
47 \\
47 \\
46.5 \\
46 \\
46 \\
46 \\
46 \\
45.8 \\
45 \\
44.8 \\
44.5 \\
44 \\
44 \\
43.5 \\
43.3 \\
43\end{array}$ & $\begin{array}{r}13 \\
12 \\
13 \\
12 \\
12 \\
11 \\
12 \\
13 \\
13 \\
12 \\
10 \\
12 \\
13 \\
13 \\
13 \\
11 \\
7 \\
12 \\
11 \\
9 \\
11 \\
10 \\
12 \\
12 \\
10 \\
9 \\
10 \\
13 \\
12 \\
12 \\
11 \\
10 \\
9 \\
12 \\
7 \\
12 \\
13 \\
12 \\
11 \\
10 \\
9 \\
9 \\
12 \\
11 \\
11 \\
11 \\
10 \\
10 \\
10 \\
9 \\
10 \\
9 \\
11 \\
10 \\
10 \\
9 \\
8 \\
7 \\
10 \\
9 \\
10 \\
10 \\
8 \\
7 \\
7 \\
7 \\
9 \\
9 \\
7 \\
8 \\
7 \\
7 \\
8 \\
8 \\
8 \\
8 \\
7 \\
7 \\
7 \\
7 \\
7\end{array}$ & 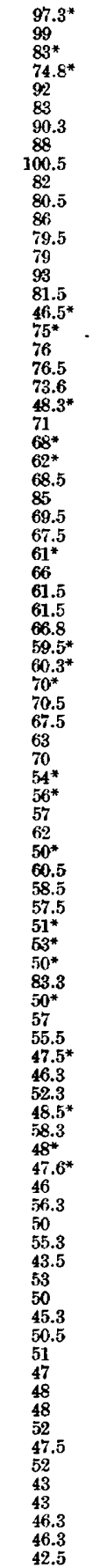 & 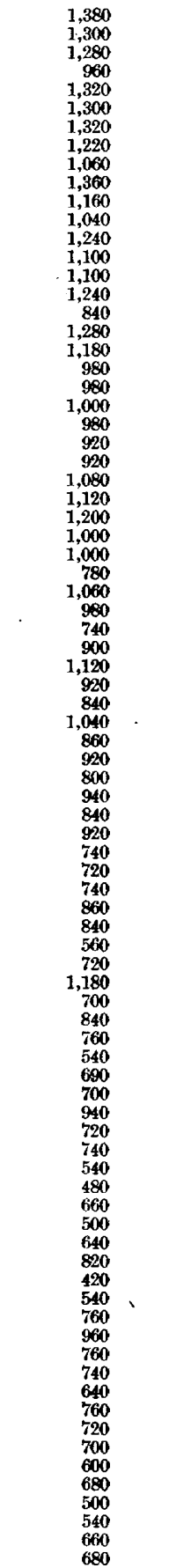 & $\begin{array}{l}1.37 \text { DB } \\
1.37 \text { DB } \\
1.248 \dagger \\
1.158 \\
1.32 \text { DB } \\
1.248 \text { DB } \\
1.29 \text { DB } \\
1.295 \\
1.37 \\
1.237 \\
1.221 \\
1.277 \\
1.212 \\
1.207 \\
1.3 \\
1.233 \\
0.845 \\
1.166 \\
1.176 \\
1.178 \\
1.153 \\
0.866 \\
1.125 \\
1.090 \\
1.026 \\
1.095 \\
1.268 \\
1.107 \\
1.085 \\
1.016 \\
1.069 \\
1.021 \\
1.021 \\
1.078 \\
0.999 \\
1.006 \\
1.114 \\
1.119 \\
1.085 \\
1.038 \\
1.114 \\
0.936 \\
0.969 \\
0.971 \\
1.026 \\
0.890 \\
1.009 \\
0.986 \\
0.976 \\
0.903 \\
0.926 \\
0.890 \\
1.25 \\
0.890 \\
0.971 \\
0.954 \\
0.858 \\
0.840 \\
0.916 \\
0.869 \\
0.984 \\
0.863 \\
0.861 \\
0.835 \\
0.961 \\
0.890 \\
0.951 \\
0.787 \\
0.926 \\
0.890 \\
0.820 \\
0.895 \\
0.903 \\
0.853 \\
0.863 \\
0.863 \\
0.913 \\
0.858 \\
0.913 \\
0.782 \\
0.782 \\
0.840 \\
0.840 \\
0.774 \\
\end{array}$ \\
\hline
\end{tabular}

* Denotes those cases falling below 7.5 per cent. normal weight for height.

$\dagger$ All surface areas, except those marked DB, are from Benedict and Talbot. DB denotes Dubois and Dubois. 
their basis. As it happened, the vital capacity of normal children and of children below normal weight for height differed very little. Those cases falling below 7.5 per cent. weight for height are marked with an asterisk.

TABLE 3.-Vital Capacity of Lungs for Boys

\begin{tabular}{|c|c|c|c|c|c|c|c|c|}
\hline Group & $\begin{array}{l}\text { Number } \\
\text { Studied }\end{array}$ & $\begin{array}{l}\text { Height, } \\
\text { Inehes }\end{array}$ & $\begin{array}{c}\text { Average } \\
\text { Vital } \\
\text { Capacity, } \\
\text { C.c. }\end{array}$ & $\begin{array}{c}\text { Surface } \\
\text { Area, } \\
\text { Square } \\
\text { Meters }\end{array}$ & $\begin{array}{l}\text { Number } \\
\text { Within } \\
20 \% \text { of } \\
\text { Normal } \\
\text { Vital } \\
\text { Capacity }\end{array}$ & $\begin{array}{c}\text { Number } \\
\text { Falling } \\
\text { Below } \\
\text { 80\% } \\
\text { Average } \\
\text { Vital } \\
\text { Capacity }\end{array}$ & $\begin{array}{c}\text { Highest } \\
\text { Vital } \\
\text { Capacity }\end{array}$ & $\begin{array}{l}\text { Lowest } \\
\text { Vital } \\
\text { Capacity }\end{array}$ \\
\hline $\begin{array}{c}\text { I } \\
\text { Total } \\
\text { Average }\end{array}$ & $\begin{array}{c}8 \\
4^{*} \\
12 \\
\ldots\end{array}$ & $\begin{array}{c}63-69.8 \\
\ldots \ldots\end{array}$ & $\begin{array}{l}2,080 \\
1,610 \\
1,845\end{array}$ & $\begin{array}{l}1.600 \\
1.344 \\
1.514\end{array}$ & 4 & $\begin{array}{l}1 \\
0\end{array}$ & $\begin{array}{l}2,290 \\
1,960\end{array}$ & $\begin{array}{l}1,600 \\
1,260\end{array}$ \\
\hline $\begin{array}{c}\text { II } \\
\text { Total } \\
\text { Average }\end{array}$ & $\begin{array}{c}50 \\
16^{*} \\
66 \\
\cdots\end{array}$ & $\begin{array}{l}57-63 \\
\ldots \ldots\end{array}$ & $\begin{array}{l}1.390 \\
1,343 \\
1,366\end{array}$ & $\begin{array}{l}1.338 \\
1.233 \\
1.313\end{array}$ & $\begin{array}{l}46 \\
15\end{array}$ & $\begin{array}{l}4 \\
1\end{array}$ & $\begin{array}{l}1,800 \\
2,240\end{array}$ & $\begin{array}{r}720 \\
1,020\end{array}$ \\
\hline $\begin{array}{c}\text { III } \\
\text { Total } \\
\text { Average }\end{array}$ & 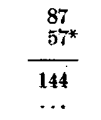 & $\begin{array}{c}51-5\} \\
\ldots \ldots\end{array}$ & $\begin{array}{l}1,100 \\
1,091 \\
1,095\end{array}$ & $\begin{array}{l}1.157 \\
1.093 \\
1.132\end{array}$ & $\begin{array}{l}79 \\
49\end{array}$ & $\begin{array}{l}8 \\
8\end{array}$ & $\begin{array}{l}1,580 \\
1,420\end{array}$ & $\begin{array}{l}620 \\
600\end{array}$ \\
\hline $\begin{array}{c}\text { IV } \\
\text { Total } \\
\text { Average }\end{array}$ & $\frac{27}{17^{*}}$ & $46-51$ & $\begin{array}{l}840 \\
814 \\
\end{array}$ & $\begin{array}{l}0.988 \\
0.917 \\
0.961\end{array}$ & $\begin{array}{l}24 \\
15\end{array}$ & $\begin{array}{l}3 \\
2\end{array}$ & $\begin{array}{l}1,100 \\
1,100\end{array}$ & $\begin{array}{l}600 \\
540\end{array}$ \\
\hline
\end{tabular}

* Denotes those cases falling below 7.5 per cent. normal weight for height.

TABLE 4.-Vital Capacity of Lungs for Girls

\begin{tabular}{|c|c|c|c|c|c|c|c|c|}
\hline Group & $\begin{array}{l}\text { Number } \\
\text { Studied }\end{array}$ & $\begin{array}{l}\text { Height, } \\
\text { Inches }\end{array}$ & $\begin{array}{c}\text { Average } \\
\text { Vital } \\
\text { Oapacity } \\
\text { O.c. }\end{array}$ & $\begin{array}{c}\text { Surface } \\
\text { Area, } \\
\text { Square } \\
\text { Meters }\end{array}$ & $\begin{array}{c}\text { Number } \\
\text { Within } \\
20 \% \text { of } \\
\text { Normal } \\
\text { Vital } \\
\text { Capacity }\end{array}$ & $\begin{array}{c}\text { Number } \\
\text { Falling } \\
\text { Below } \\
80 \% \\
\text { Average } \\
\text { Vital } \\
\text { Capacity }\end{array}$ & $\begin{array}{c}\text { Highest } \\
\text { Vital } \\
\text { Oapacity }\end{array}$ & $\begin{array}{c}\text { Lowest } \\
\text { Vital } \\
\text { Capacity }\end{array}$ \\
\hline I & 26 & $59.5-53$ & $\begin{array}{l}1,120 \\
1,025\end{array}$ & $\begin{array}{l}1.204 \\
1.052\end{array}$ & $\begin{array}{r}24 \\
8\end{array}$ & $\begin{array}{l}2 \\
0\end{array}$ & $\begin{array}{l}1,380 \\
1,280\end{array}$ & $\begin{array}{l}740 \\
840\end{array}$ \\
\hline $\begin{array}{c}\text { Total } \\
\text { Average }\end{array}$ & $\begin{array}{l}34 \\
\ldots\end{array}$ & $\ldots$. & 1,072 & 1.168 & & & & \\
\hline II & $\begin{array}{l}22 \\
14^{*}\end{array}$ & $53-47$ & $\begin{array}{l}759 \\
786\end{array}$ & $\begin{array}{l}0.976 \\
0.900\end{array}$ & $\begin{array}{l}18 \\
11\end{array}$ & $\begin{array}{l}4 \\
3\end{array}$ & $\begin{array}{l}1,180 \\
1,120\end{array}$ & $\begin{array}{l}420 \\
540\end{array}$ \\
\hline $\begin{array}{c}\text { Total } \\
\text { Average }\end{array}$ & $\begin{array}{c}36 \\
\ldots\end{array}$ & $\cdots \cdot$ & 773 & 0.938 & & & & \\
\hline III & 14 & $47-43$ & 693 & 0.849 & 12 & 2 & 960 & 500 \\
\hline
\end{tabular}

* Denotes those cases falling below 7.5 per cent. normal weight for height.

The tables used for surface area are the work of Benedict and Talbot ${ }^{6}$ who kindly allowed us to use these tables before publication. ${ }^{7}$ For the heavier children we used the chart of Dubois and Dubois. ${ }^{8}$

6. Talbot, F., and Benedict, F. G.: The Metabolism and Growth from Birth to Puberty, Carnegie Institution, Washington; Publication 302.

7. These figures are available now in Carnegie Publication No. 302.

8. Dubois, D., and Dubois, E. F.: Arch. Int. Med. 17:863 (June) 1916. 
Our readings of the vital capacity for boys and girls are shown in Tables 1 and 2 . These readings were divided into four groups for boys and three groups for girls (Tables 3 and 4 ). The difference in the height between the shortest and tallest children in the case of boys was 23.8 inches and of the girls 16.5 inches. Accordingly, we allowed six inches roughly for each group. It will be noticed that the first group for boys is a little over six inches and the last group for boys is a little under six inches. The third group of girls is from 43 to 47.1 inches, a division made necessary by the small number of girls of the shortest height.

In each group the average vital capacity is given first for children of normal height and weight. Below this figure the vital capacity is given for those falling below 7.5 per cent. of the average weight for height.

The next column is the average surface area for each group. The figures for surface area are given in square meters and those for vital capacity in cubic centimeters. In order to compare the two, the vital capacity can be read in litres.

Table 5.-Summary and Ratio of Vital Capacity to Surface Area

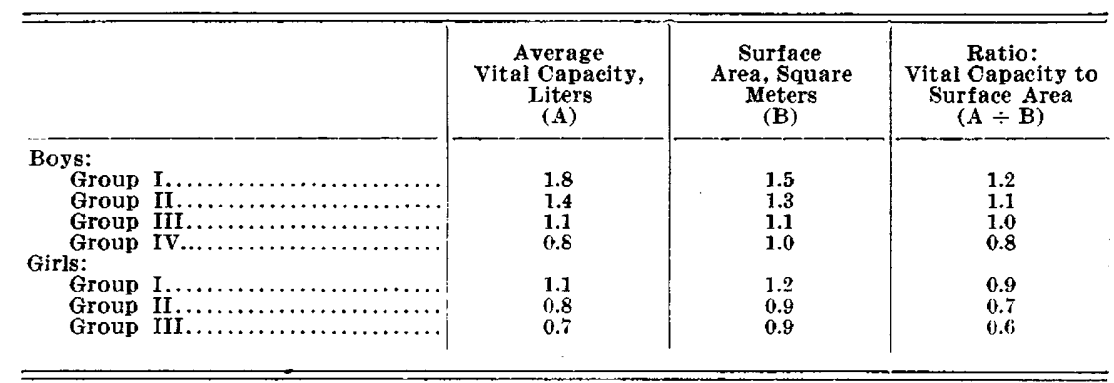

For the second group of boys, which contained next to the largest number, the vital capacity in liters would be 1.4, and the surface area 1.3 square meters. For the third group, which contained the largest number of boys, the vital capacity in liters was 1.1 , and the surface area 1.1 square meters. These two groups show very markedly the close relationship between vital capacity and surface area. Even in the first group with comparatively few determinations the vital capacity was 1.8 liters and the surface area 1.5 square meters.

The next column in the tables gives the number of cases in which the vital capacity fell below 20 per cent. of the average for this group: a variation of 10 per cent. in adults is considered normal. Children, as a rule, are more difficult to handle and are usually harder to make cooperate, so that they vary more from the average than adults. A variation of 20 per cent. includes most of the cases.

The next column in the tables gives the number of cases in which vital capacity fell below 20 per cent. of the average for this group. 
The next column gives the number below 80 per cent. of the average; not 80 per cent. below, but below 80 per cent. of the average.

In pathologic conditions there is a fall in vital capacity. From our determinations the fall should amount to more than 20 per cent. of the average to be considered significant.

The highest and lowest vital capacity is given for each group. This shows a wide variation of about 20 per cent. from the average.

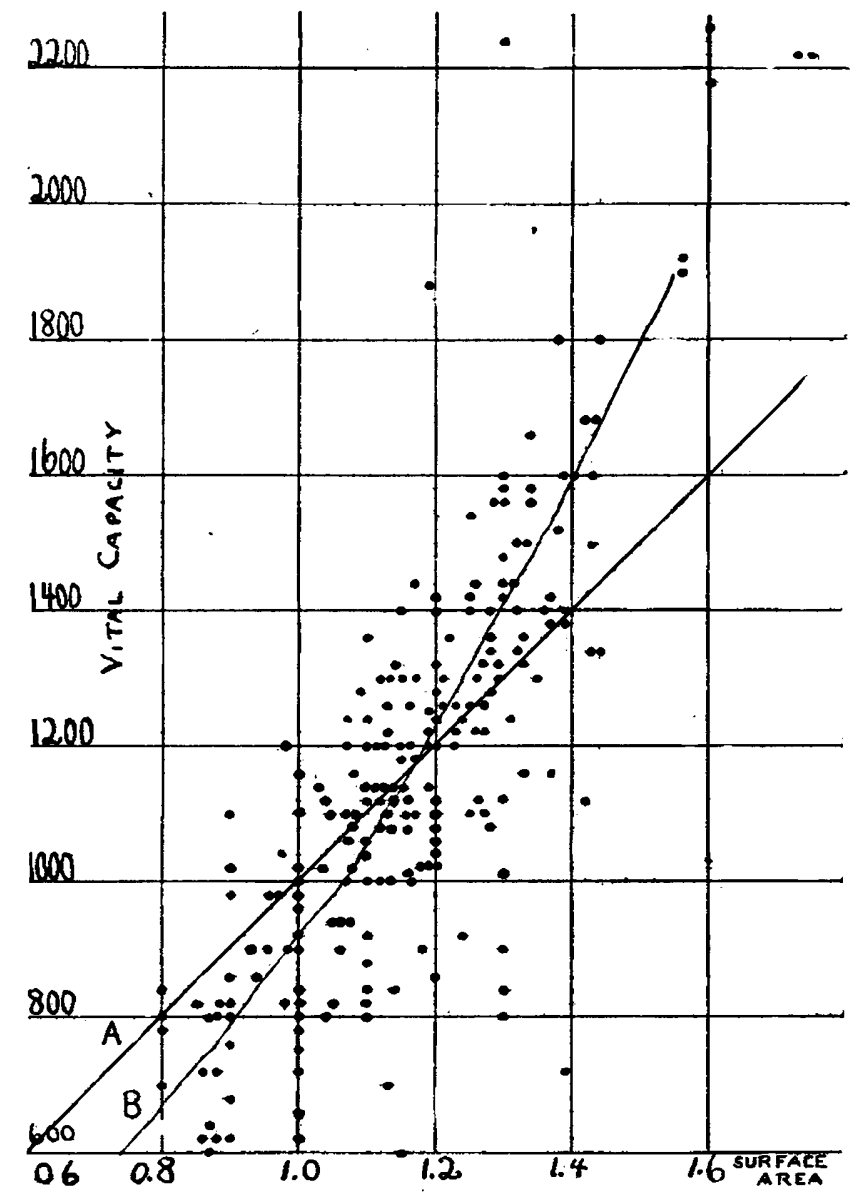

A.-When vital capacity and surface area are identical theoretically. B.--Experimental Curve.

Group 1 of the girls gave an average vital capacity of 1.1 liters and a surface area of 1.2 square meters. Group II of the girls gave an average vital capacity of 0.8 liters and a surface area of 0.9 square meters.

In Table 5 we have summarized the vital capacity in liters, the surface area in square meters, and the ratio of vital capacity to surface 
area for each group-first of boys, then of girls. Group I of our girls, our best group of girls, showed a vital capacity of I liter to 0.9 of a square meter of surface area; and our best group of boys, Group III, showed a vital capacity of 1 liter to 1 square meter of surface area.

Although the normal children and those falling below 7.5 per cent. of normal were considered separately, the vital capacity showed very little variation; in fact, they corresponded very closely, except in the first group of boys, which was a very small one.

On a graph, a line was drawn arbitrarily to make the vital capacity and surface area correspond exactly. We then plotted our actual figures and found that they came very close to this arbitrary line.

\section{CONCLUSIONS}

1. A very close relationship was found between vital capacity and surface area in children.

2. There is practically no difference between children considered in the tables for normal weight for height and those falling 7.5 per cent. below the normal.

3. The determinations of vital capacity for girls were on the whole much lower than those for boys, but the same relation held between vital capacity and surface area.

4. Determinations of vital capacity below seven years of age are unreliable. 